\title{
Frequency Response modeling of Active Filters
}

\author{
Aaron Don M. Africa, Adrian Co, Nathan Delgado, Jose Medalla, Aaron Sarmiento, Elijah Tesalona \\ Department of Electronics and Communications Engineering \\ De La Salle University, Manila \\ 2401 Taft Ave., Malate, Manila 1004, \\ Philippines, aaron.africa@dlsu.edu.ph
}

\begin{abstract}
It is expected from this paper that Active filters will be discussed in detail and strengthened with examples. There will be explanations on the different types of this filter, and also the different passes, which will show what the signal will or will not have. The concept and theory behind the filters will also be discussed, as to give a proper introduction to filters. Data concerning the differences among these types will be evidence to prove and demonstrate how the filters can affect the signal in different situations. Lastly, the paper will explain how a filter is used and how it works. The numerous applications behind filters and how they affect signals will show how important they can be to clean and remove unnecessary parts of said signal. Hence, the paper will be separated in a certain way, with the initial half explaining the general information behind it, and the second half explaining in further detail and giving some data to enhance the envision of the filter.
\end{abstract}

Key words: Active, Filter, Butterworth, Elliptic, Bessel, Chebyshev.

\section{INTRODUCTION}

Active filters make use of Amplifiers to create a more efficient performance and allow a logical outcome when the filler is used. A kind of filter that is composed of active components of a circuit is an active filter. Four types of fillers shall be discussed in this paper. They are Butterworth, Chebyshev, Bessel, and Elliptical [1,2]. The paper shall denote the differences in their purpose, and how each purpose can affect how filler is used. Furthermore, the applications of the active filters will also be discussed, to emphasize how this can affect certain situations. A look at the hands-on approach will allow teaching one how to utilize these functions and apply the information in the possible projects one may do $[3,4,5]$.

Active Filters suppress noise so that the signal received will have certain parts of it cut off, allowing a cleaner signal to pass, without the 'noise'. Generating such a signal will filter out the parts not within certain conditions, hence creating a smaller signal to work with. Depending on the filter used, the parts of the signal taken out will be different. The differences in each filter will be made apparent in the paper's discussion.
Learning these concepts is important since the application will not make as much sense unless they learn them [6].

Now the reason why differentiating them is that there are also passes, namely, high pass, bandpass, and low pass. They are used in the filter types as a means to measure the signal's values and apply it to different kinds. As their name suggests, the high pass and low pass filter focus on signals that are lower or higher at a certain point. Bandpass on the other hand makes use of a range and will either include the range, or disregard it $[7,8]$. The paper will focus on all of these, and give further information pertaining to filters and their many applications.

\section{BACKGROUND OF THE STUDY}

In approximately 1890, the improvement of long-distance transmission lines with the use of coils and regular intervals was sought by numerous people. For a long time, there were no results and advancements to reach that goal until 1899 when M.I. Pupin studied about these cables. He discovered that a line that has coils at regular intervals can be replaced by an equivalent uniform cable with properly spaced coils. The measurement of the gap connecting the two adjacent coils is inversely proportional to the equivalence. The equivalence vanishes completely when the gap is bigger than half of the measurement of the wavelength of the signal. Through his keen study, Pupin discovered that we can significantly decrease the damping of telegraphy and telephony cables through the proper installment of the coils. They named it Pupin lines and it became famous all over the world [9].

The characteristics of these Pupin lines were studied by George A. Cambell and found out that Pupin lines have a frequency-dependent effect. There is a certain frequency called critical frequency that changes the damping qualities. The space between the coils establishes the critical frequency which is closely similar to a wavelength that is equivalent to a doubled distance in the middle of them. When the frequency is lower than the critical frequency the damping is low and dependent on the losses of cable. Contrastingly, the opposite effect happens when the frequency is higher than the critical frequency. Campbell noticed that this effect can be efficiently used for removing harmonics in signal generators. Also, he used it to make a cable that can be a lowpass filter and open the possibilities for having a cable that utilized as a bandpass 
filter through combining coils and capacitors. In 1914, filters were born when Karl Willy Wagner and Campbell both but independently tested a circuit with resistors, capacitors, and inductors that are constructed in a ladder pattern. The filters they have created have studied or developed by numerous scientists like Otto J. Zobel, S. Butterworth, Sidney Darlington, M. Reed, etc. Each study of the filter has helped in every development of it. Aside from the first and last elements, the other elements in a Campbell filter are the same but when Butterworth was developing it, they can already change some elements. It is now being used in different technologies for various industries. For example, the filter is now being used as an RF filter that is applicable in communications and the medical industry [10].

\section{STATEMENT OF THE PROBLEM}

One of the tasks engineers are sure to encounter is creating circuits. However, in using this source, we would also be getting some noise in the output frequency in the AC Current. So, we use filters to filter out the signals we get from the source. Aiming for a signal without any noise would be a goal for us to create a clean signal without unnecessary values. Similar to other fields, computer engineers must aim for precision and efficiency [11]. Having such discipline is also necessary for other projects, hence, having the patience and tenacity to properly complete these tasks will allow us to evolve into better problem solvers. Learning more about these applications will allow us to learn which filtration method will be best for a certain time. Becoming flexible is also another value that aspiring engineers must have and evolve [12].

\section{SIGNIFICANCE OF THE STUDY}

As we have researched earlier, we as a group have picked up significant areas of interest and discussion with regards to grabbing information about the nature of filters and its practical uses in the industry, or for consumer use. Our goal as researchers is to make people appreciate the usefulness of these certain types of electronics, mainly because this can aid with their tasks in the profession that they are in. For example, a biomedical researcher can use active filters to interface psychological sensors to undertake diagnostics and data logging. With that being said, the performers of this experiment must realize the usefulness while interacting with this said equipment, as this may be used in the long run once they are in their profession. Moreover, we got to learn as well whilst researching this particular topic, more so with its applications. We would not be aware of such applications if we did not get to pay attention to the importance of these concepts. It is an eye-opener for the many people that go into sound engineering since they use these concepts about their profession [13]. It is also a delight that most of these filters are used for practical reasons, mainly because it is possible to collect and refine some frequencies with the use of low-pass filter method as seen from this circuit as shown in Figure 1:

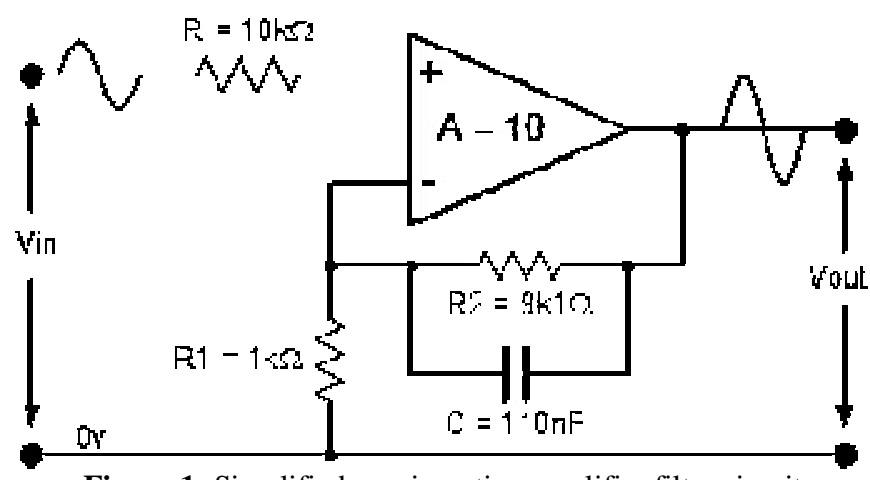

Figure 1: Simplified non-inverting amplifier filter circuit

\section{DESCRIPTION OF THE SYSTEM}

There are four types of active filters, each producing a unique frequency response. Each filter shares a similar roll-off slope of $-40 \mathrm{~dB} /$ decade when attaining frequencies larger than its respective cut off points. The Butterworth filter also called the maximally flat or flat filter, produces a flat frequency response curve until it reaches its cut off frequency. As James Andrews said, "The Butterworth is sometimes referred to as the 'Maximally Flat Amplitude' Filter,'. Among all types, this filter displays the sharpest attenuation due to its non-linear phase-shift to frequency function. It also displays a flat frequency gain both within and below the cut off region and shares middling characteristics between the Chebyshev and Bessel filters. It exhibits a non-linear phase response along with a moderate skirt roll-off.

The Chebyshev filter also called an equal ripple filter, utilizes a sharper cut off compared to the Butterworth filter in the passband. Similarly, it also displays a large phase shift at a cut off frequency. It is flexible in the sense that its maximum and minimum gains below the cutoff point can be adjusted by the designer. The filter exhibits larger pear to peak ripples at faster roll-off speeds while having a highly non-linear phase response in the skirt region. It is commonly used when the user requires a very sharp roll-off at the expense of a gain ripple at lower frequencies. A Chebyshev filter also displays a highly non-linear response frequency curve which causes delays of data frequency in the passband. This in turn may cause serious pulse distortions which increases the number of errors for modern demodulators. Thus, users of the Chebyshev filter often increase the filter bandwidth, allowing for an extension of the phase region.

The Bessel filter displays a linear phase response with ideal characteristics until its cut off frequency with a mild skirt slope. Due to its ideal phase characteristics especially concerning its constant group delay, it is often used in situations where this is required such as analog video signal processing. It has a flat-time delay that is optimal. It utilizes minimal phase shifts while being most suitable for pulse applications.

Lastly, the elliptic filter is used for applications that require a very high attenuation for quick transitions between the 
stopband and passband. It is most commonly seen in RF applications such as industrial remote controls or wireless alarm home systems. It utilizes the sharpest roll-off among all filters discussed in the transition region. This causes ripples in both the passband and stopband regions as seen in the graph below. Due to its high attenuation, the other frequencies included in the stopband are reduced [14].

One who needs a filter can choose among these types and according to, filter order, the cost of the filter, difficulty of tuning, ripples are some of the basis for choosing the correct type of filter to use. Figure 2 shows the Frequency Response Curve.

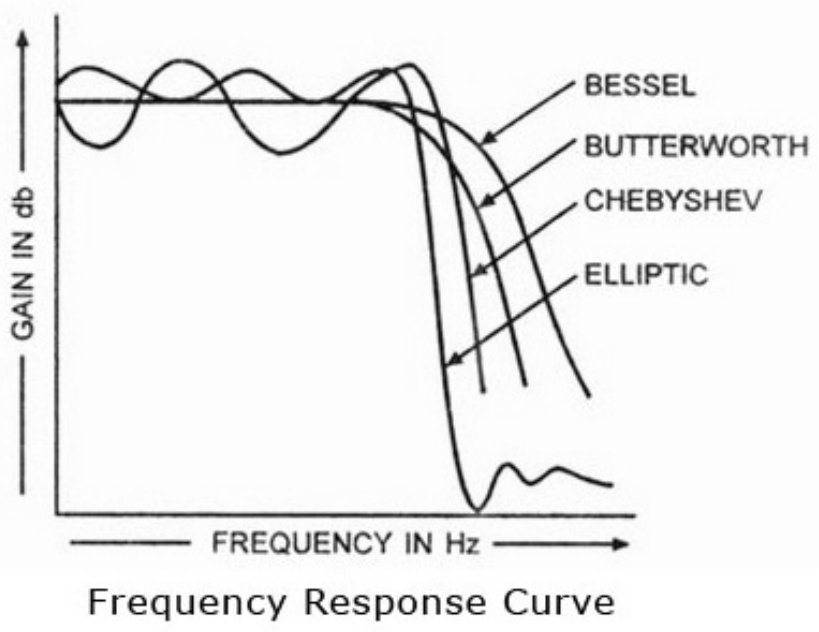

Figure 2: Frequency response curve

\section{METHODOLOGY}

\subsection{Research Method}

An extensive literature review will be conducted by the group to achieve the objective of discussing how filters work and how can they be applied so that people from different industries can benefit from it. This method is chosen since all the data the students need already exist and therefore they just have to research for the data. No scientific experiments were conducted by the group since there is no hypothesis being tested and no new scientific information trying to be established. Even though there will be no new academic discoveries, an extensive literature review will help the students to reach their goal of collecting useful information about the functions and applications of filters and sharing that information with people from different industries. This information will be shared with the readers will hopefully help them appreciate the science behind the function of the filters and help them look for ways to apply it in their industry.

\subsection{Data Gathering and Analysis}

Data will be gathered by looking for numerous academic sources like research papers and educational websites and collecting the important information that we can get from these sources to help us achieve our objective. Specifically, we will research information about how filters work and in what technologies are they applied to. Data will be analyzed by understanding what kind of filters are used in the technologies we have researched and how essential are they to make the technologies work.

\section{THEORETICAL CONSIDERATIONS}

Active filters are considered to be part of almost any circuit theory, by which its specialty is to be used in an op-amp. By definition, it is a universal active filter by which it can be configured for a range of low-pass, high-pass, and bandpass filters. In theory, it should at least suppress some signals to ensure that the signal that is passing through has been silenced or toned down to ensure the clarity of such signal or audio being processed and listened. With the filters at play, it is also important to note that it is useful to utilize these filters, mainly because it specifies frequencies to ensure that the frequency that is desired is being utilized while creating a certain circuit or output. Active filters on a more specific note come in so many shapes and sizes, and most of these active filters comprise namely: Butterworth, Chebyshev, and Bessel filter. These active filters act as equal ripple filters, flat filters, cutoff frequency filters to name a few.

For the first classification of an active filter, namely the Butterworth filter, it serves as a flat filter. There is a certain flat response that surfaces whilst the active filter is powered on, and that area in the graph depicted from the filter is called the brick wall response. The gain usually at the brick wall response area is at 0 , hence dictating the purpose of such filter, which is to flatten some frequencies. As stated in the graph, there are certain cut-off frequencies, all by which display different downward slopes as the frequencies get higher and higher over time, and upon testing of this certain range of frequencies. Figure 3 shows the graph of the Butterworth filter.

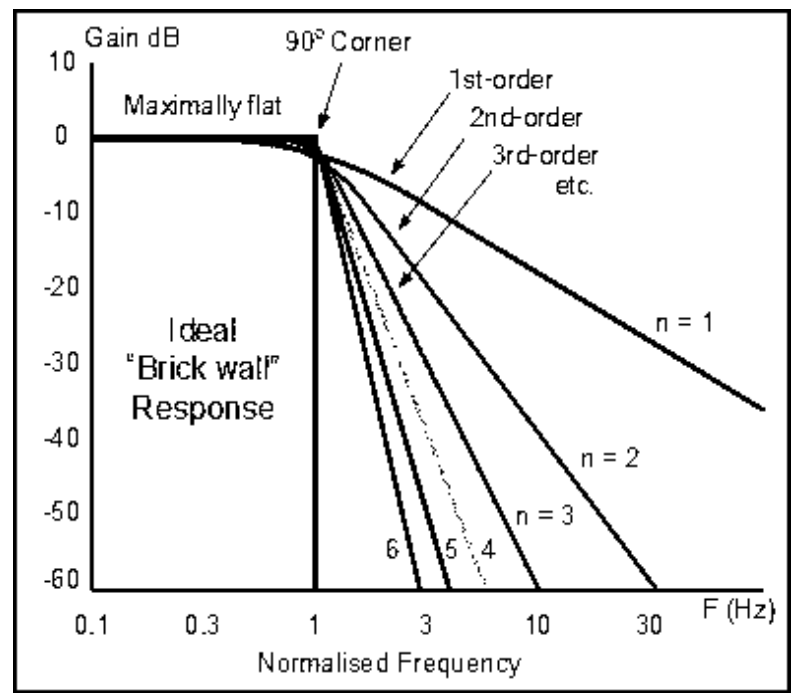

Figure 3: Butterworth filter 
The purpose of this filter when applying this in a practical sense would be shaping the frequency spectrum of such signal, by which there would be communication and controls system involved in the duration of the signal being filtered over time. Since there are orders involved in the process of running the said filter, it would then output the best signal since there are stages of refining these said signals. Decades and octaves are also involved, by which it is needed to divide the frequencies into segments to further refine what needs to be refined from those segments. In theory, the logarithmic frequency scale determines the decades and octaves needed when you desire to refine those signals set at the frequencies starting from the scale shown in Figure 4.

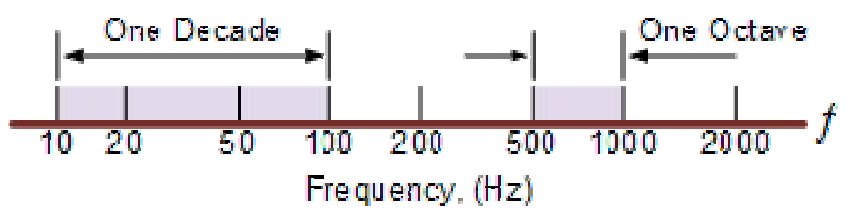

Figure 4: Logarithmic frequency scale

Such software like freeware that uses this particular is the application called Engineers Filter, and this filter plugin that allows for extremely steep filtering. The user can demo such signals the user may wish to apply a filter to, and judging from the application, it can also showcase different filters for application such as Chebyshev, and Cauer to name a few, by which it can display in more detail the gist of lowpass, highpass, and bandpass modulation waveforms.

For the second classification of active filters, there is the Chebyshev Filter and this specific filter is also called an equal ripple filter. The cutoff region as seen from the graph below is much more defined and that it gives off a sharper cutoff region than compared to the Butterworth filter in its passband. Although this type of filter can be effective for practical uses, its disadvantage over the other filters would be the exterior of the gain, as indicated by its minima and maxima in the said graph that is stated below. All of the graph's values are merely expressed in decibels $(\mathrm{dB})$. Figure 5 shows the graph of the Chebysev filter.

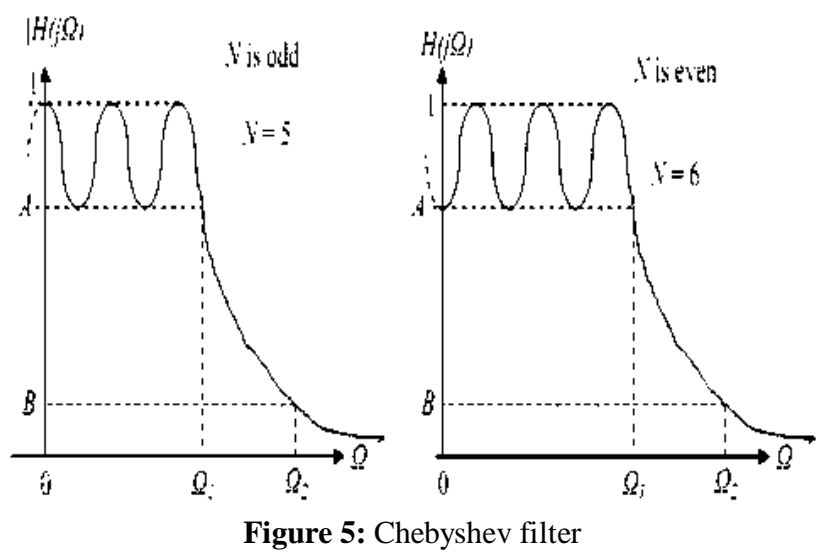

What is more striking about this filter compared to the previous filters, would be that this kind of filter is completely stable compared to the other ones mentioned. This kind of filter can exhibit stable propagation over time, making this the most effective filter out of them all, since this filter will require less effort to process such signals and/or frequencies [15]. For example, if you will pass a square wave through this filter, then it will also output a square wave which will not exceed beyond the desired frequency, ensuring that the process of processing these signals will live up to its name of being stable as the certain signal passes through the Bessel filter as shown in figure 6 .

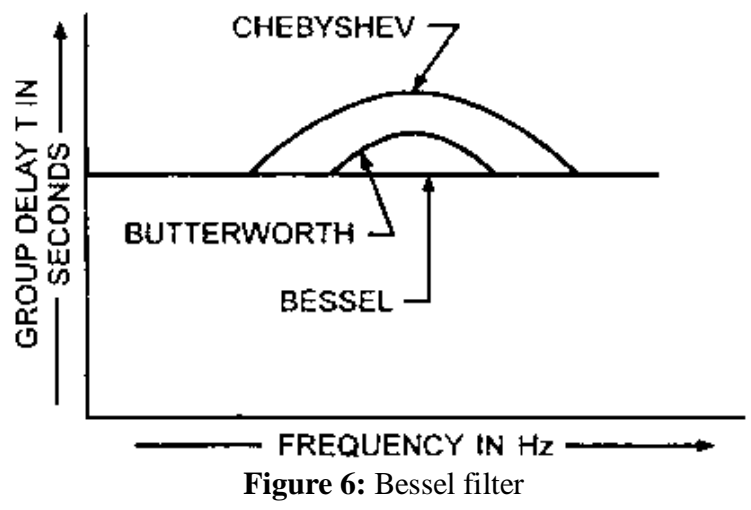

\section{DATA AND RESULTS}

Upon further research, it can be found that active filters may be typically found in technologies that require an enhancement in both predictability and performance. It commonly acts as an amplifier without the use of inductors. It contains electronic components such as resistors and capacitors, while op-amps are used to allow for the easier utilization of filters. When comparing them to passive filters, it is more suitable to apply active ones due to the use of smaller components in its apparatus. The main use of active filters is for noise suppression and to isolate a signal to achieve clear communication. Analog active filters are used in audio systems to send various frequencies to different speakers. Recording and playback systems implement active filters to control the frequency signals. Biomedical instruments also use these filters specifically diagnostic equipment for physiological sensors.

Butterworth filters are used to achieve a perfect fit in the passband region due to its flat response and high roll-off frequency curve. It is most commonly applied in audio processing systems for its easy implementation and characteristics which include a sensible roll-off and a slightly non-linear phase response. It also acts as a filter for a signal's frequency spectrum in communication systems and control systems such as voicemail devices, air conditioners, and refrigerators. The Butterworth filter's maximally flat passband characteristic allows it to be an anti-aliasing filter in data converter applications. Other examples that use this type of filter are high-quality audio applications, digital motion analysis, and radar target track display. 
The Chebyshev filter on the other hand is not used in audio applications due to its ripple characteristics in the passband despite a steeper roll-off. It is commonly applied in applications that allow only one frequency in the passband while requiring other frequencies to eliminate noise. It operates explicitly as an analog or digital filter which reduces the errors in actual or ideal filters. Chebyshev filters are most likely used in RF applications requiring a steep transition between passband and stopband to remove unwanted products such as the intermodulation of harmonics.

The Bessel filter is applied in systems which require ideal phase characteristics from its filter. It is often used in pulse applications for this purpose while utilizing its small phase shift characteristic. Its stable propagation delay across the $\mathrm{i} / \mathrm{p}$ frequency spectrum will allow a square frequency wave in the $\mathrm{o} / \mathrm{p}$ when a similarly square wave is received in the input. This is useful for digital systems that require fast transitions such as clock generators for microprocessors. Devices that implement complex control systems such as pulse width control systems for audio and radio applications also use the Bessel filter.

Lastly, the Elliptical filter is used in many RF applications which require a very fast transition between passband and stopband is required. Examples of these applications include remote controls, wireless home security systems, and wireless meter reading.

\section{ANALYSIS OF DATA}

Based on the data presented, there are four different types of filters, each with their own unique use. We have the Butterworth filter which filters out the unnecessary noise in the signal, Chebyshev Filter that is known to be correct and reduce errors in transmissions, Bessel Filters that are known to be able to transition between sudden changes in signals and Elliptical Filters that is known to be used in fast paced transmission applications.

\section{CONCLUSION}

As stated, the paper has explained in detail how the active filters work, with the concepts and theories sufficiently discussed. The filters discussed in the paper have been thoroughly explained, with an analysis and an expounded discussion regarding them. A rich review of the many fields that can apply with filters allowed this paper to have several evidences that the filters have applications that must be learned, to further improve the ongoing times. While it may be difficult to achieve, the many researchers that could be seen discussing these filters is a sign that the research will continue, and be able to reach more heights, if focused on. The paper that has been discussed should allow awareness to spread that these filters aren't as simple as they seem and can become complicated as one goes on.

In summary, the Active Filter is used as a means to produce a cleaner signal. By having a signal with as little noise as possible, the data transmitted will be clearer rather than cryptic. While the four filters have a different use, all of them focus on filtering a certain part of the signal. Knowing the difference will be better, while also applying for the proper pass. Errors are unavoidable, so it's best to filter or remove them. These filters allow the signal to do as such, so learning their concepts can help in real-life applications. Being in several fields, and not only electronics, but these filters also can be flexible and provided people with further alternatives in both the created and to be created alternatives.

The different filters and the ways to use them are important to understand their importance. If one studies them more thoroughly, it can be possible to implement their knowledge, and create further inventions or improve.

\section{RECOMMENDATIONS}

There are some things to point out whilst making this paper, one of which would be the time that we have allotted while making the paper, mainly because that is our main gripe, and that we had a hard time collecting our thoughts while making the paper. Although it was hectic for us to compress all information into one paper, we got to pick up the important topics of discussion, such as the usefulness of the filters in real-life situations. As computer engineers, it is important that we are aware that these concepts and equipment that support these concepts exists, mainly because we would not get to appreciate what crisp audio sounds like without the application of low-pass, high-pass, and bandpass filters, which is in the form of the active filters mentioned above.

The utmost reason why we chose this topic is because of its complexity, in which we find the beauty behind it, but we felt like our explanations and research should have been improved in terms of comprehensibility of the material/s that we, as a team, have researched over the course of making this paper. We would like to mention the references that have helped us in the curation of this paper, as this has helped us fully understand the nature of these filters, and only if we were given more time to do some research, we would get to interact with different references to broaden our research.

\section{REFERENCES}

[1] J. García-Niebla RN and G. Serra-Autonell MD, "Effects of inadequate low-pass filter application," Journal of Electrocardiology. Vol. 42, No. 4, pp.303-304, 2009.

[2] S. Mahata, S. KumarSaha, R. Kar and D. Mandal, "Optimal design of fractional order low pass Butterworth filter with accurate magnitude response," Digital Signal Processing. Vol. 72, pp. 96-114, 2018.

[3] X.L.Guo, C.W.Sun, Z.H.Bao, C.Xu, G.Zhang, Z.L.Wang, H.H.Yin, X.F.Zhang and H.Jiang, "Tunable low-pass Ka-band MEMS filter based on Electromagnetic-Bandgap Structure," Sensors and Actuators A: Physical. Vol. 247, pp. 83-89, 2016. https://doi.org/10.1016/j.sna.2016.05.028

[4] J.Bingi, M.Hemalatha, R.W.Anita, C.Vijayan and V.M.Murukeshan, "Asymmetric transmission and optical 
low-pass filtering in a stack of random media with graded transport mean free path," Optical Materials. Vol. 49, pp. 15-20, 2015.

[5] A. Hurst, S. Goodman, J. Hilton and J. VanDeWeert, "Miniature low-pass mechanical filter for improved frequency response with MEMS microphones \& low-pressure transducers," Sensors and Actuators A: Physical. Vol. 210, pp. 51-58, 2014.

[6] J. Xu, Z. Li, L. Liu, C. Chen, B. Xu, P. Ning and C. Gu, "Low-pass plasmonic filter and its miniaturization based on spoof surface plasmon polaritons," Optics Communications. Vol. 372, pp. 155-159, 2016.

[7] M. Numada, T. Nomura, K. Yanagi, K. Kamiya and H. Tashiro, "High-order spline filter and ideal low-pass filter at the limit of its order," Precision Engineering. Vol. 31, No. 3, pp. 234-242, 2007. https://doi.org/10.1016/j.precisioneng.2006.09.002

[8] L. Cao, H. Li and Y. Zhang, "Retinal image enhancement using low-pass filtering and $\alpha$-rooting," Signal Processing.Vol. 170, 2020.

[9] R. Kulkarni and P. Rastogi, "Simultaneous unwrapping and low pass filtering of continuous phase maps based on autoregressive phase model and wrapped Kalman filtering," Optics and Lasers in Engineering. Vol. $124,2020$.

[10] R. Verma, N. Pandey and R. Pandey, "CFOA based low pass and high pass fractional step filter realizations," AEU - International Journal of Electronics and Communications. Vol. 99, pp. 161-176, 2019.

[11] A. Africa, P.Arevalo, A.Publico, and M.Tan, "Digital control systems functions and applications," International Journal of Advanced Trends in Computer Science and Engineering. Vol. 8, No. 4, pp. 1368-1371, 2019. https://doi.org/10.30534/ijatcse/2019/52842019

[12] B. Singh, A. K. Tiwari and S. K. Agrawal,"State variable-based tow thomas biquad filter using GM-C universal design using 180nm CMOS technology," International Journal of Advanced Trends in Computer Science and Engineering. Vol. 9, No. 2,pp. 1882-1887, 2020.

https://doi.org/10.30534/ijatcse/2020/149922020

[13] A. AbdelAty, A. Soltan, W. Ahmed and A. Radwan, "Fractional order Chebyshev-like low-pass filters based on integer order poles," Microelectronics Journal. Vol. 90,pp. 72-81, 2019.

[14] K. RanjanJha and M. Rai, "Modification in microstrip low pass filter using bulb shape patch," AEU International Journal of Electronics and Communications. Vol. 63, No. 12, pp. 1076-1079, 2009.

[15] M. Knoll, M. Uther and A. Costall,"Effects of low-pass filtering on the judgment of vocal affect in speech directed to infants, adults and foreigners," Speech Communication. Vol. 51, No. 3, pp. 210-216, 2009.

https://doi.org/10.1016/j.specom.2008.08.001 